09,08

\title{
Дефекты и некоторые физические свойства номинально чистых и легированных цинком кристаллов ниобата лития
}

\author{
(C) Н.А. Теплякова, М.В. Смирнов, Н.В. Сидоров, М.Н. Палатников \\ Институт химии и технологии редких элементов и минерального сырья им. И.В. Тананаева \\ ФИЦ „Кольский научный центр РАН“, \\ Апатиты, Россия \\ E-mail: n.tepliakova@ksc.ru
}

Поступила в Редакцию 25 февраля 2021 г.

В окончательной редакции 30 марта 2021 г.

Принята к публикации 30 марта 2021 г.

\begin{abstract}
Методами ИК-спектроскопии поглощения в области валентных колебаний $\mathrm{OH}^{-}$-групп, фотолюминесценции в видимой области спектра, фотоиндуцированного рассеяния света исследованы особенности дефектной структуры и их влияние на свойства кристаллов $\mathrm{LiNbO}_{3}: \mathrm{Zn}$, легированных в широком концентрационном диапазоне, включающем два концентрационных порога (при $\sim 3.0$ и $\sim 6.8 \mathrm{~mol} \% \mathrm{ZnO}$ в расплаве). В кристаллах $\mathrm{LiNbO}_{3}: \mathrm{Zn}(0.004-2.01 \mathrm{~mol} \% \mathrm{ZnO})$ при увеличении концентрации цинка наблюдается увеличение концентрации гидроксильных групп и уменьшение интенсивности люминесценции от центров свечения, связанных с дефектами $\mathrm{Nb}_{\mathrm{Li}}$. Последнее, по-видимому, связано с образованием вблизи дна зоны проводимости мелких энергетических уровней при вытеснении атомами цинка атомов ниобия с позиций лития идеальной структуры и, соответственно, уменьшением концентрации дефектов $\mathrm{Nb}_{\mathrm{Li}}$. В сильнолегированных кристаллах $\mathrm{LiNbO}_{3}: \mathrm{Zn}(4.46-6.5 \mathrm{~mol} . \% \mathrm{ZnO})$ и в кристалле $\mathrm{LiNbO}_{3 \text { stoich }}\left(6.0 \mathrm{wt} . \% \mathrm{~K}_{2} \mathrm{O}\right)$ наблюдается заметно меньшая концентрация $\mathrm{OH}^{-}$-групп, увеличение ширины запрещенной зоны на $0.3-0.4 \mathrm{eV}$, увеличение интенсивности люминесценции в зеленой области спектра за счет образования новых каналов рекомбинации по сравнению со слаболегированными кристаллами. Кроме того, в таких кристаллах наблюдается увеличение протонной проводимости за счет увеличения концентрации межузельного водорода $\mathrm{H}^{+}$и, как следствие, образования множества мелких акцепторных уровней вблизи потолка валентной зоны.
\end{abstract}

Ключевые слова: ниобат лития, прямое легирование, валентные колебания $\mathrm{OH}^{-}$-групп, центры свечения, фотовольтаическое и диффузионное поля.

DOI: 10.21883/FTT.2021.08.51167.036

\section{1. Введение}

Монокристалл ниобата лития $\left(\mathrm{LiNbO}_{3}\right)$ сочетает в себе хорошие нелинейно-оптические, сегнетоэлектрические и фоторефрактивные свойства, которые определяют его широкое применение в качестве функционального оптического материала в различных устройствах электронной техники [1-3]. Ниобат лития является гетеродесмической фазой переменного состава с широкой областью гомогенности на фазовой диаграмме. Монокристалл $\mathrm{LiNbO}_{3}$ любого состава характеризуется сложным спектром многочисленных дефектов различного вида, равновесных и неравновесных $[1,4]$. Благодаря особенностям его кислородно-октаэдрической структуры, в которой только две трети октаэдров могут быть заполнены основными и примесными катионами, а треть октаэдров остаются вакантными, состояние дефектности и физические характеристики кристалла $\mathrm{LiNbO}_{3}$ можно изменять в широких пределах путем легирования и изменения стехиометрии (отношения $R=\mathrm{Li} / \mathrm{Nb}$ ) $[1,4]$. При этом особенности локализации в структуре комплексных дефектов, обусловленных присутствием в кристалле гидроксильных групп, часто являются определяющими для получения оптических материалов высокой степени структурного совершенства [5].
Эффект фоторефракции („optical damage“) существенно ограничивает применение кристаллов $\mathrm{LiNbO}_{3}$ для преобразования и модуляции лазерного излучения. Для создания высокосовершенных оптических материалов с низким эффектом фоторефракции, высокой композиционной и оптической однородностью актуальны исследования изменения дефектной структуры кристалла $\mathrm{LiNbO}_{3}$ при легировании его „нефоторефрактивными“ катионами металлов $\left(\mathrm{Mg}^{2+}, \mathrm{Zn}^{2+}, \mathrm{In}^{3+}, \mathrm{Sc}^{3+}\right.$ и др.), понижающими эффект фоторефракции за счет увеличения фотопроводимости [6-8]. Наиболее эффективное подавление эффекта фоторефракции достигается при легировании ниобата лития магнием или цинком при концентрациях, близких ко второму концентрационному порогу $(\sim 5.5 \mathrm{~mol} . \% \mathrm{MgO}$ и $\sim 6.8 \mathrm{~mol} . \% \mathrm{ZnO}$ в расплаве) [8]. При концентрациях легирующей добавки вблизи концентрационных порогов, как правило, существенно понижается композиционная однородность кристалла ниобата лития и повышается его общая дефектность. В то же время, может заметно уменьшаться концентрация отдельных видов заряженных дефектов, оказывающих заметное влияние на практически значимые свойства кристалла, такие как стойкость к оптическому повреждению. Использование цинка в качестве легирующей добавки в некоторых случаях более предпо- 
чтительно, так как он позволяет более существенно, чем магний изменять состояние дефектности, ширину запрещенной зоны, расположение в ней энергетических уровней, а также достигать большей композиционной и оптической однородности кристаллов ниобата лития [9]. Это преимущество является особенно важным для получения высокосовершенных оптических кристаллов ниобата лития с высоким уровнем легирования, близким к пороговому значению.

В данной работе методами ИК-спектроскопии поглощения в области валентных колебаний $\mathrm{OH}^{-}$-групп, фотолюминесценции в видимой области спектра, фотоиндуцированного рассеяния света (ФИРС) исследованы особенности дефектной структуры и влияние дефектов на фоторефрактивные свойства серии кристаллов $\mathrm{LiNbO}_{3}$ легированных цинком в широком диапазоне концентраций: $\mathrm{Zn}(0.004-6.5 \mathrm{~mol} \% \mathrm{ZnO})$, включающем концентрационные пороги при $\sim 3.0$ и $\sim 6.8 \mathrm{~mol} . \% \mathrm{ZnO}$.

\section{2. Методика эксперимента}

Кристаллы выращены методом Чохральского в воздушной атмосфере на установке „Кристалл-2“ из гранулированной шихты ниобата лития, синтезированной в ИХТРЭМС КНЦ РАН [10]. Кристалл конгруэнтного состава $\left(\mathrm{LiNbO}_{3 \text { cong }}\right)$ получен из конгруэнтного расплава $(\mathrm{Li} / \mathrm{Nb}=0.946)$. Выращивание стехиометрического кристалла $\left(\mathrm{LiNbO}_{3 \text { stoich }}\left(6.0\right.\right.$ wt.\% $\left.\left.\mathrm{K}_{2} \mathrm{O}\right)\right)$ осуществлялось по технологии HTTSSG (High temperature top seeded solution growth technique) из расплава конгруэнтного состава $(\mathrm{Li} / \mathrm{Nb}=0.946$ в шихте) в присутствии щелочного растворителя $\mathrm{K}_{2} \mathrm{O}\left(\approx 6.0\right.$ wt.\%). Кристаллы $\mathrm{LiNbO}_{3}: \mathrm{Zn}$ (0.004-6.50 mol.\% ZnO) получены методом прямого легирования [9]. Концентрация следовых количеств посторонних примесей в кристаллах не превышает $1 \cdot 10^{-3}$ для $\mathrm{Mo}, \mathrm{Fe}, \mathrm{Ti}, \mathrm{Si}, \mathrm{Pb}, \mathrm{Ni}, \mathrm{Cr}, \mathrm{Co}$ и $5 \cdot 10^{-4}$ wt\% для $\mathrm{Al}, \mathrm{Cu}, \mathrm{Mn}, \mathrm{V}, \mathrm{Mg}, \mathrm{Sn}$. Все кристаллы были монодоменизированы методом высокотемпературного электродиффузионного отжига при охлаждении со скоростью $20^{\circ} \mathrm{C} / \mathrm{h}$ в температурном интервале от $\sim 1240-890^{\circ} \mathrm{C}$ в условиях приложенного постоянного напряжения [9]. Контроль степени монодоменности кристаллов осуществлялся электроакустическим методом, основанным на анализе частотной зависимости электрического импеданса, и путем определения величины статического пьезомодуля $\left(d_{333 \mathrm{cт}}\right)$ кристаллической були [9].

Образцы для исследований вырезались в виде прямоугольных параллелепипедов с размерами $\sim 8 \times 7 \times 6 \mathrm{~mm}^{3}$, ребра которых совпадали по направлению с кристаллографическими осями $X, Y, Z(Z-$ полярная ось кристалла). Грани параллелепипедов тщательно полировались.

Регистрация спектров ИК-поглощения производилась с помощью спектрометра IFS $66 \mathrm{v} / \mathrm{s}$ фирмы Bruker при возбуждении линией $514.5 \mathrm{~nm}$ мощностью $150 \mathrm{~mW}$ аргонового лазера ILA-120. Определения объемной концентрации $\mathrm{OH}^{-}$-групп в исследованных кристаллах проводилась с помощью методики Клавира [11]. Для обработки контуров спектральных линий были использованы пакеты программ: Origin 8.1, производства фирмы Origin Lab-Corporation, LabSpec 5.5, Bomem Grammes V. 2.03. Спектры фотолюминесценции регистрировались в 90-градусной геометрии с объема исследуемых образцов с помощью спектрографа SOL SL-100M с ПЗСдетектором FLI ML 1107 BlackIlluminated (Hamamatsu) в диапазоне длин волн 380-750 nm в нормальных условиях. В качестве источника возбуждения люминесценции использовался непрерывный $\mathrm{He}-\mathrm{Cd}$ лазер $\left(\lambda_{\mathrm{exc}}=325 \mathrm{~nm}, 15 \mathrm{~mW}\right)$. Из каждого фотолюминесцентного спектра вычитался фоновый сигнал. Методика определения величины фотоэлектрических полей в исследованных кристаллах по параметрам индикатрисы спекл-структуры ФИРС подробно описана в работе [12].

\section{3. Результаты и обсуждение}

На рис. 1 представлены ИК-спектры поглощения в области валентных колебаний $\mathrm{OH}^{-}$-групп, на рис. 2 спектры фотолюминесценции, на рис. 3 - кар-

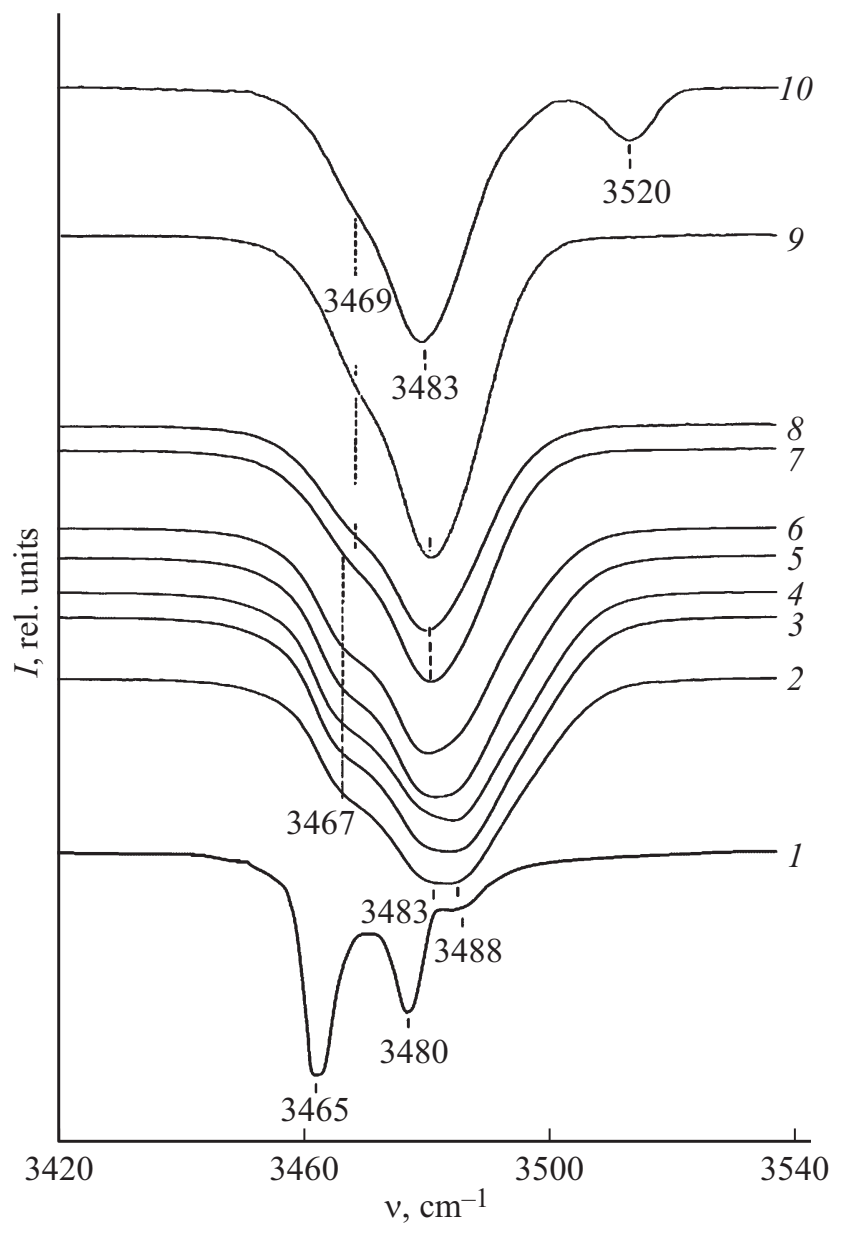

Рис. 1. Спектры ИК-поглощения в области валентных колебаний $\mathrm{OH}^{-}$-групп кристаллов: $1-\mathrm{LiNbO}_{3 \text { stoich }}\left(6.0 \mathrm{wt} \% \mathrm{~K}_{2} \mathrm{O}\right)$; $2-\mathrm{LiNbO}_{3 \text { cong }} ; 3-\mathrm{LiNbO}_{3}: \mathrm{Zn}(0.004) ; 4-\mathrm{LiNbO}_{3}: \mathrm{Zn}$ (0.07); $5-\mathrm{LiNbO}_{3}: \mathrm{Zn}(1.19) ; 6-\mathrm{LiNbO}_{3}: \mathrm{Zn}(1.40) ; 7-$ $\mathrm{LiNbO}_{3}: \mathrm{Zn}(2.01) ; 8-\mathrm{LiNbO}_{3}: \mathrm{Zn}(4.46) ; 9-\mathrm{LiNbO}_{3}: \mathrm{Zn}$ (4.54); $10-\mathrm{LiNbO}_{3}: \mathrm{Zn}(6.50 \mathrm{~mol} . \% \mathrm{ZnO})$. 


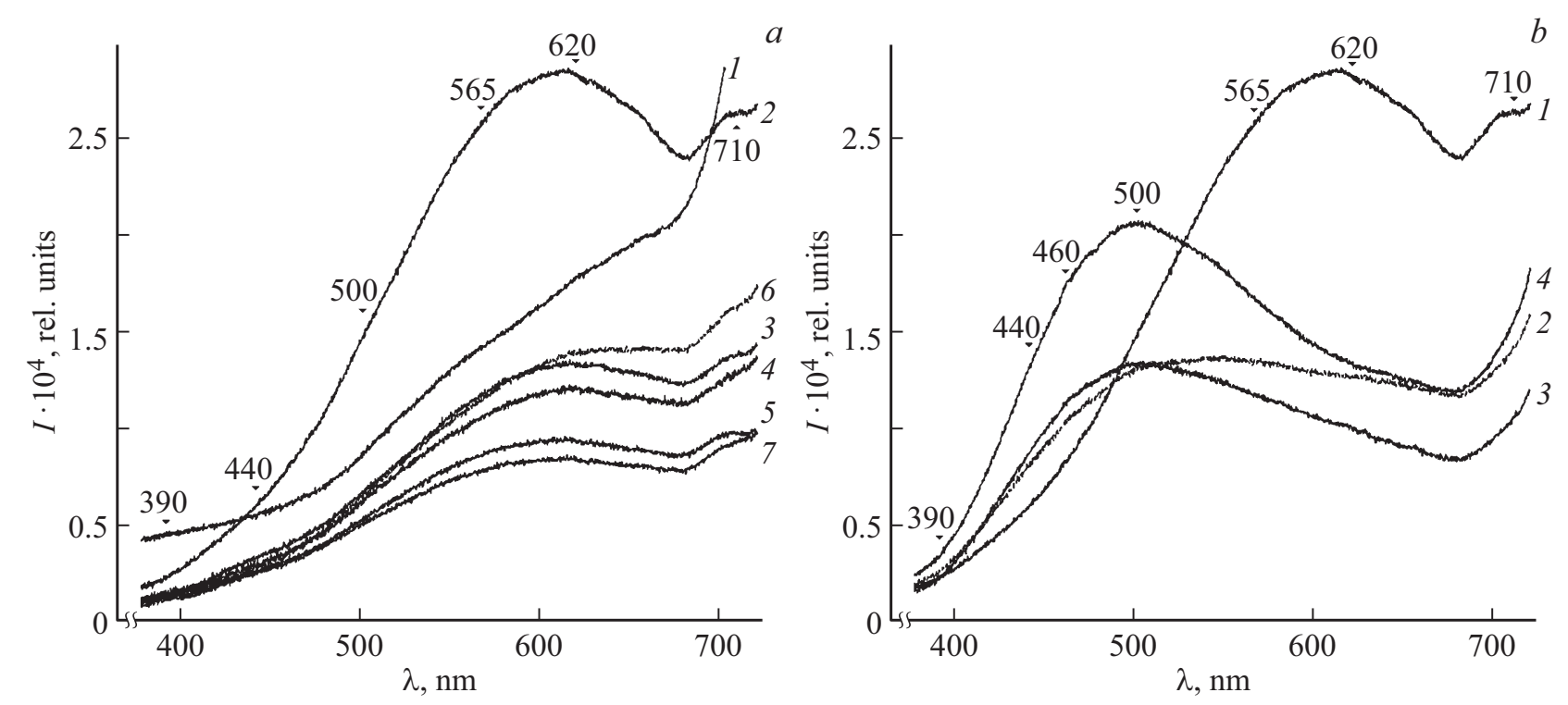

Рис. 2. Спектры фотолюминесценции кристаллов ниобата лития: a) $1-\mathrm{LiNbO}_{3 \mathrm{stoich}}\left(6.0 \mathrm{wt} \% \mathrm{~K}_{2} \mathrm{O}\right)$, $2-\mathrm{LiNbO}_{3 \mathrm{cong}}$, $3-\mathrm{LiNbO}_{3}: \mathrm{Zn}(0.004), 4-\mathrm{LiNbO}_{3}: \mathrm{Zn}(0.07), 5-\mathrm{LiNbO}_{3}: \mathrm{Zn}(1.19), 6-\mathrm{LiNbO}_{3}: \mathrm{Zn}(1.40), 7-\mathrm{LiNbO}_{3}: \mathrm{Zn}(2.01) ;$ b) $1-\mathrm{LiNbO}_{3 \text { cong, }} 2-\mathrm{LiNbO}_{3}: \mathrm{Zn}(4.46) ; 3-\mathrm{LiNbO}_{3}: \mathrm{Zn}(4.54) ; 4-\mathrm{LiNbO}_{3}: \mathrm{Zn}(6.50 \mathrm{~mol} . \% \mathrm{ZnO})$.
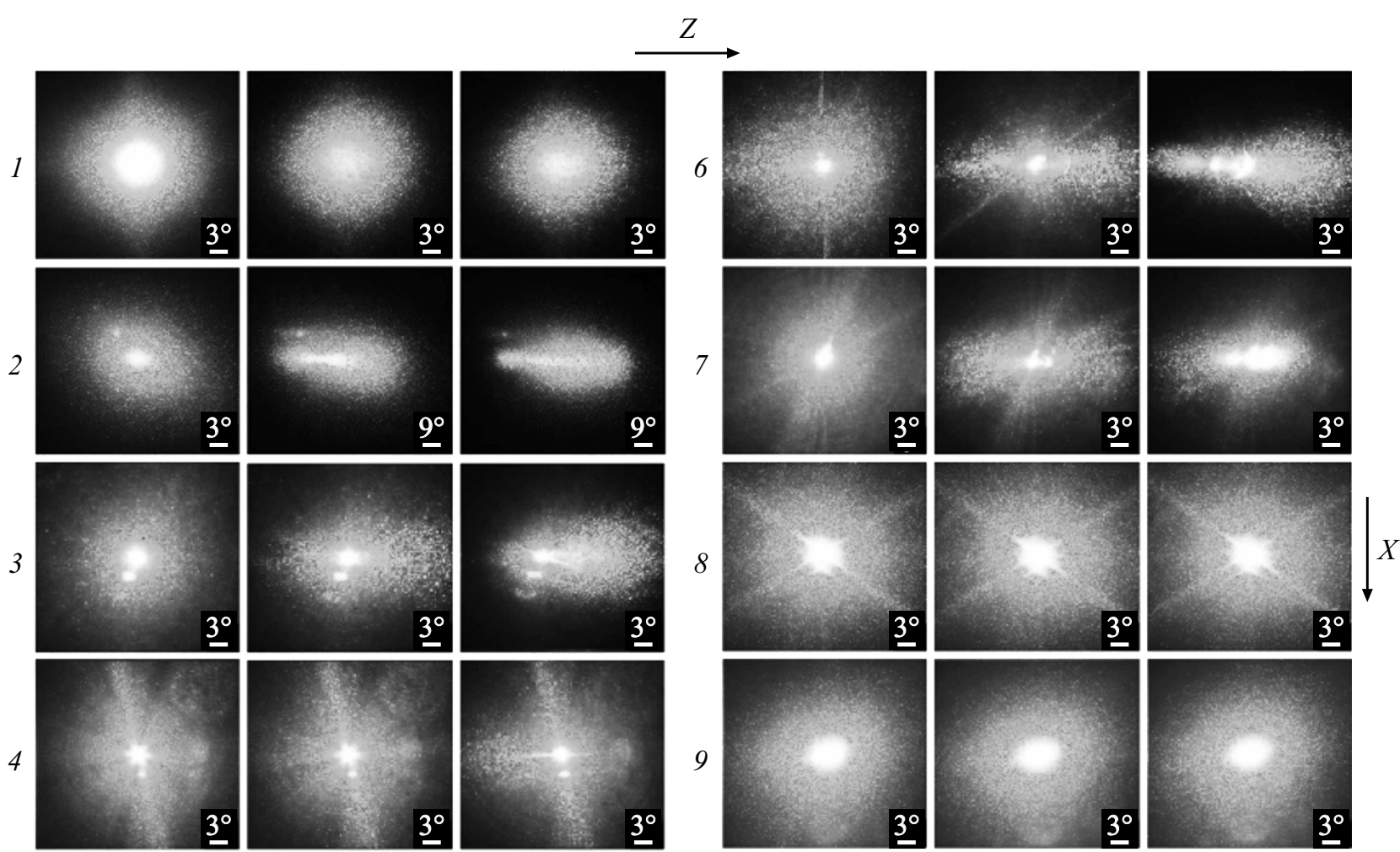

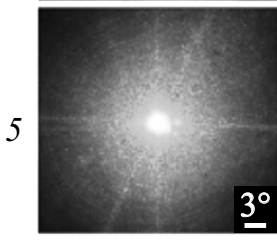

$1 \mathrm{~s}$

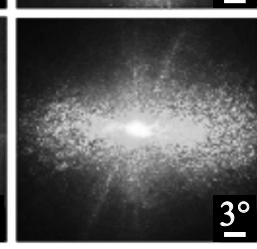

$30 \mathrm{~s}$

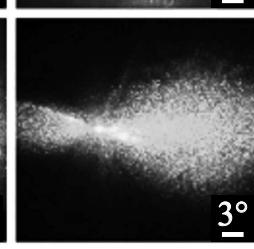

$360 \mathrm{~s}$

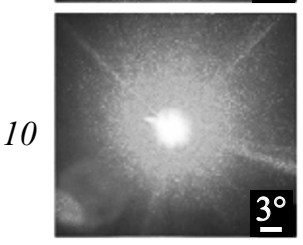

$1 \mathrm{~s}$

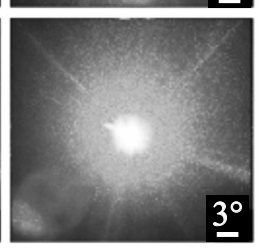

$30 \mathrm{~s}$

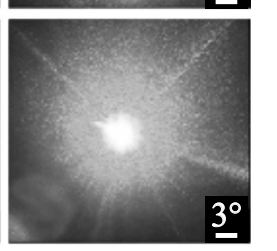

$360 \mathrm{~s}$

Рис. 3. Картины ФИРС кристаллов: $1-\mathrm{LiNbO}_{3 \text { cong }} ; 2-\mathrm{LiNbO}_{3 \text { stoich }}\left(6.0 \mathrm{wt} \% \mathrm{~K}_{2} \mathrm{O}\right) ; 3-\mathrm{LiNbO}_{3}: \mathrm{Zn}(0.004) ; 4-\mathrm{LiNbO}: \mathrm{Zn}$ (0.07); $5-\mathrm{LiNbO}_{3}: \mathrm{Zn}(1.19) ; 6-\mathrm{LiNbO}_{3}: \mathrm{Zn}(1.40) ; 7-\mathrm{LiNbO}_{3}: \mathrm{Zn}(2.01) ; 8-\mathrm{LiNbO}_{3}: \mathrm{Zn}(4.46) ; 9-\mathrm{LiNbO}: \mathrm{Zn}(4.54) ;$ $10-\mathrm{LiNbO}_{3}: \mathrm{Zn}(6.50 \mathrm{~mol} . \% \mathrm{ZnO}) . \lambda_{0}=532 \mathrm{~nm} . P=160 \mathrm{~mW}$. 
тины ФИРС кристаллов $\mathrm{LiNbO}_{3 \text { stoich }}\left(6.0 \mathrm{wt} . \% \mathrm{~K}_{2} \mathrm{O}\right)$, $\mathrm{LiNbO}_{3 \text { cong }}$ и $\mathrm{LiNbO}_{3}: \mathrm{Zn}(0.004-6.5 \mathrm{~mol} \% \mathrm{ZnO})$. Видно, что для кристаллов разного состава полученные данные заметно отличаются. Для объяснения наблюдаемых отличий необходимо рассмотреть тонкие особенности сложной дефектной структуры исследованных кристаллов $\mathrm{LiNbO}_{3}$, как гетеродесмической фазы переменного состава.

Дефекты $\mathrm{Nb}_{\mathrm{Li}}$ (катионы $\mathrm{Nb}^{5+}$ находящиеся в позициях катионов $\mathrm{Li}^{+}$идеальной структуры стехиометрического состава) в кристаллах $\mathrm{LiNbO}_{3 \text { stoich }}\left(6.0 \mathrm{wt.} \% \mathrm{~K}_{2} \mathrm{O}\right)$, $\mathrm{LiNbO}_{3 \text { cong }}$ и $\mathrm{LiNbO}_{3}: \mathrm{Zn}$ являются глубокими электронными ловушками $[4,13]$, образующими при захвате электронов, поляроны малого радиуса $\left(\mathrm{Nb}_{\mathrm{Li}}^{3+}\right)$ и биполяроны $\left(\mathrm{Nb}_{\mathrm{Li}}^{3+}-\mathrm{Nb}_{\mathrm{Nb}}^{4+}\right)$, которые обеспечивают осуществление поляронной проводимости вплоть до температуры $\sim 400 \mathrm{~K}[13,14]$. В конгруэнтном кристалле $\mathrm{LiNbO}_{3}$ количество дефектов $\mathrm{Nb}_{\mathrm{Li}}$ составляет примерно $\sim 1 \mathrm{~mol} \%$ [15]. В идеальном стехиометрическом кристалле $(R=1)$, в котором идеальный порядок чередования катионов вдоль полярной оси $(\mathrm{Li}, \mathrm{Nb}$, вакантный октаэдр), дефекты $\mathrm{Nb}_{\mathrm{Li}}$ отсутствуют. В реальных кристаллах $\mathrm{LiNbO}_{3}$, по составу близких к стехиометрическим, концентрация дефектов $\mathrm{Nb}_{\mathrm{Li}}$ очень мала. Дефекты $\mathrm{Nb}_{\mathrm{Li}}$ имеют эффективный заряд $+4|e|$, где $e-$ заряд электрона, по отношению к решетке. Компенсация заряда для выполнения условия электронейтральности осуществляется другими точечными дефектами, в частности, дефектами $\left(\mathrm{V}_{\mathrm{Li}}\right)$ - вакансиями в положении катионов $\mathrm{Li}^{+}$идеальной структуры стехиометрического состава [4]. Дефекты $\mathrm{V}_{\mathrm{Li}}$ - преобладающие точечные дефекты в структуре нелегированного конгруэнтного кристалла $\mathrm{LiNbO}_{3}$. Они являются дырочными ловушками с энергией захвата дырки $-0.44 \mathrm{eV}$ [16]. Согласно модели компенсации дефектов $\mathrm{Nb}_{\mathrm{Li}}$ литиевыми вакансиями $\left(\mathrm{V}_{\mathrm{Li}}\right)$ [4], концентрацию точечных дефектов $\mathrm{V}_{\mathrm{Li}}$ и $\mathrm{Nb}_{\mathrm{Li}}$ в кристаллической решетке реальных беспримесных кристаллов $\mathrm{LiNbO}_{3 \text { stoich }}\left(6.0 \mathrm{wt} \% \mathrm{~K}_{2} \mathrm{O}\right)$ и $\mathrm{LiNbO}_{3 \text { cong }}$ можно рассчитать по формулам [17]:

$$
\begin{gathered}
C\left(\mathrm{~V}_{\mathrm{Li}}\right)=100 \times\left(\frac{4-4 \mathrm{Li} / \mathrm{Nb}}{5+\mathrm{Li} / \mathrm{Nb}}\right), \\
C\left(\mathrm{Nb}_{\mathrm{Li}}\right)=C\left(\mathrm{~V}_{\mathrm{Li}}\right) / 4 .
\end{gathered}
$$

Для расчета концентрации дефектов $\mathrm{V}_{\mathrm{Li}}$ и $\mathrm{Nb}_{\mathrm{Li}}$ в структуре сильно легированных кристаллов $\mathrm{LiNbO}_{3}: \mathrm{Zn}(4.46-6.50 \mathrm{~mol} . \% \mathrm{ZnO})$ можно, использовались формулы, предложенные в работе [17]:

$$
\begin{gathered}
C\left(\mathrm{~V}_{\mathrm{Li}}\right)=100 \times\left(\frac{1-\mathrm{Li} / \mathrm{Nb}}{2}\right), \\
C\left(\mathrm{Nb}_{\mathrm{Li}}\right)=0 .
\end{gathered}
$$

Из формул (1-4) видно, что для расчетов концентрации точечных дефектов $\mathrm{V}_{\mathrm{Li}}$ и $\mathrm{Nb}_{\mathrm{Li}}$ необходимо знать отношение $R=\mathrm{Li} / \mathrm{Nb}$ в кристалле $\mathrm{LiNbO}_{3}$. Определить

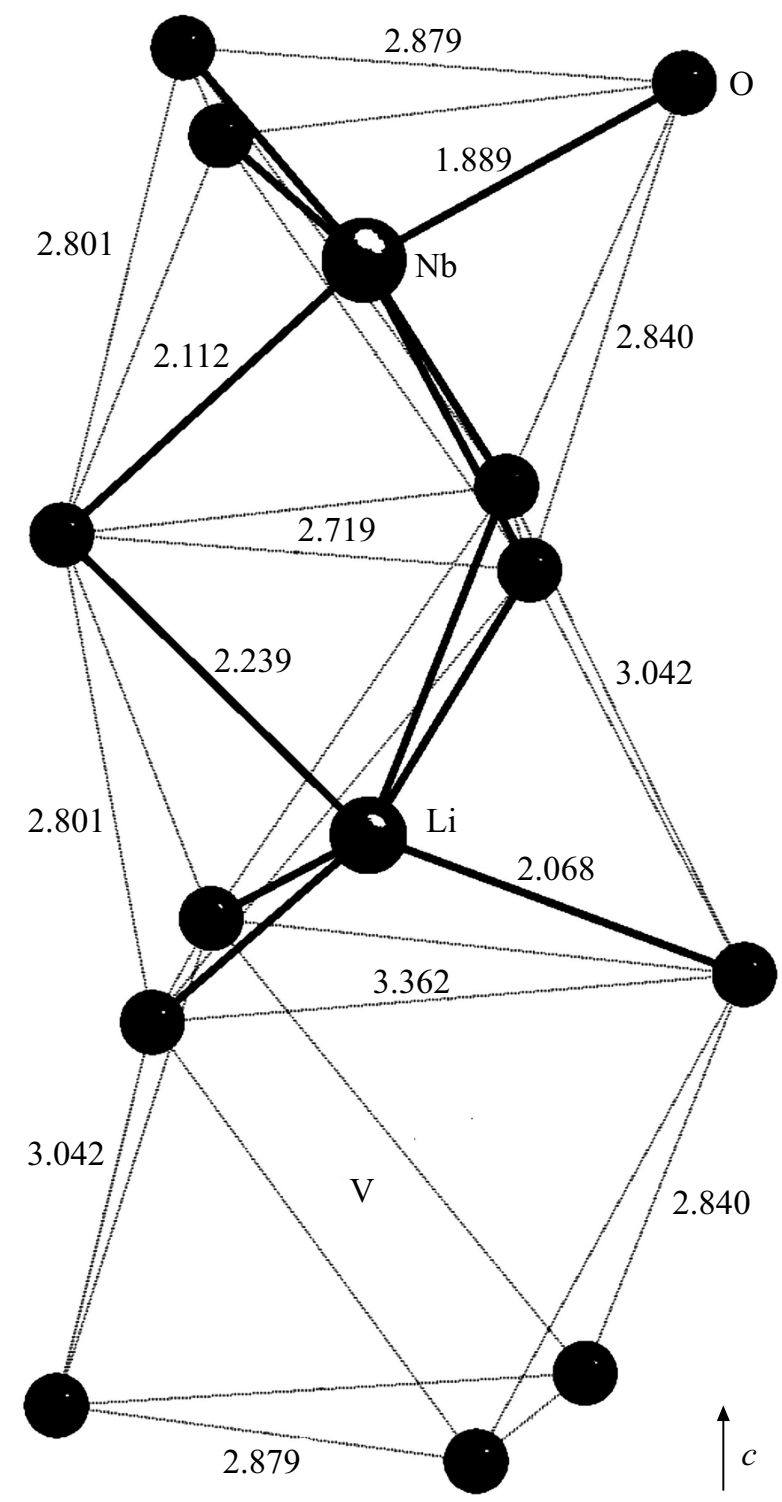

Рис. 4. Кислородно-октаэдрические кластеры $\mathrm{NbO}_{6}, \mathrm{LiO}_{6} \mathrm{VO}_{6}$ структуры кристалла $\mathrm{LiNbO}_{3}$.

величину $R$ можно по ИК-спектрам поглощения в области валентных колебаний $\mathrm{OH}^{-}$-групп. Кристалл $\mathrm{LiNbO}_{3}$ состоит из не эквивалентных друг другу кислороднооктаэдрических кластеров $\mathrm{NbO}_{6}, \mathrm{LiO}_{6}, \mathrm{Nb}_{\mathrm{Li}} \mathrm{O}_{6}, \mathrm{~V}_{\mathrm{Li}} \mathrm{O}_{6}$ и др. (рис. 4), в которых, к тому же, атомы кислорода связаны с атомами водорода водородной связью. Наличие водородной связи приводит к образованию в кристалле $\mathrm{LiNbO}_{3}$ комплексных дефектов различного типа $\left(\mathrm{Nb}_{\mathrm{Li}}-\mathrm{OH}, \mathrm{V}_{\mathrm{Li}}-\mathrm{OH}\right.$ и др). При этом локализация в структуре $\mathrm{LiNbO}_{3}$ атомов водорода определяется составом кристалла, а также способом его легирования [9]. Такая особенность структуры гетеродесмического кристалла $\mathrm{LiNbO}_{3}$ позволяет определить величину $R=\mathrm{Li} / \mathrm{Nb}$ по ИК-спектрам поглощения в области валентных колебаний $\mathrm{OH}^{-}$-групп $\left(3400-3550 \mathrm{~cm}^{-1}\right)$ по величине отноше- 
Фотоэлектрические параметры ФИРС, ширина запрещенной зоны, отношение $\mathrm{Li} / \mathrm{Nb}$, концентрация $\mathrm{OH}^{-}$-групп $\left(C(\mathrm{OH}) / \mathrm{cm}^{-3}\right)$, количество точечных дефектов $\mathrm{Nb}_{\mathrm{Li}}$ и $\mathrm{V}_{\mathrm{Li}}$ в кристаллах ниобата лития при $T_{k}$

\begin{tabular}{l|c|c|c|c|c|c|c|c}
\hline \multicolumn{1}{c|}{ Кристалл } & $\begin{array}{c}E, \\
\mathrm{eV}\end{array}$ & $\begin{array}{c}E_{P V}, \\
\mathrm{kV} / \mathrm{cm}\end{array}$ & $\begin{array}{c}E_{D}, \\
\mathrm{kV} / \mathrm{cm}\end{array}$ & $\begin{array}{c}\theta, \\
\circ\end{array}$ & $\begin{array}{c}\mathrm{V}_{\mathrm{Li}}, \\
\mathrm{mol} . \%\end{array}$ & $\begin{array}{c}\mathrm{Nb}_{\mathrm{Li}}, \\
\mathrm{mol} \%\end{array}$ & $\mathrm{Li} / \mathrm{Nb}$ & $\begin{array}{c}C(\mathrm{OH}), \\
\mathrm{cm}^{-3}\end{array}$ \\
\hline $\mathrm{LiNbO}_{3 \text { stoich }}\left(6.0 \mathrm{wt} \% \mathrm{~K}_{2} \mathrm{O}\right)$ & 3.379 & 6.855 & 2.508 & 46 & 0.60 & 0.15 & 0.991 & $4.03 \cdot 10^{16}$ \\
\hline${ }^{*} \mathrm{LiNbO}_{3 \text { cong }}$ & 3.780 & 6.232 & 0.81 & - & 3.64 & 0.91 & 0.946 & $8.15 \cdot 10^{16}$ \\
\hline $\mathrm{LiNbO}_{3}: \mathrm{Zn}(0.004)$ & 3.685 & 4.82 & 1.256 & 18 & 3.24 & 0.81 & 0.952 & $1.52 \cdot 10^{17}$ \\
\hline${ }^{*} \mathrm{LiNbO}_{3}: \mathrm{Zn}(0.07)$ & 3.659 & 6.075 & 0.65 & - & 4.88 & 1.22 & 0.928 & $1.55 \cdot 10^{17}$ \\
\hline $\mathrm{LiNbO}_{3}: \mathrm{Zn}(1.19)$ & 3.609 & 4.73 & 2.313 & 23 & 2.68 & 0.67 & 0.960 & $1.65 \cdot 10^{17}$ \\
\hline $\mathrm{LiNbO}_{3}: \mathrm{Zn}(1.40)$ & 3.626 & 8.203 & 1.159 & 16.5 & 2.16 & 0.54 & 0.968 & $1.44 \cdot 10^{17}$ \\
\hline $\mathrm{LiNbO}_{3}: \mathrm{Zn}(2.01)$ & 3.632 & 4.783 & 1.384 & 20 & 2.16 & 0.54 & 0.968 & $1.58 \cdot 10^{17}$ \\
\hline${ }^{*} \mathrm{LiNbO}_{3}: \mathrm{Zn}(4.46)$ & 4.001 & 6.551 & 0.491 & - & 3.60 & 0 & 0.928 & $2.66 \cdot 10^{16}$ \\
\hline${ }^{*} \mathrm{LiNbO}_{3}: \mathrm{Zn}(4.54)$ & 3.953 & 6.823 & 0.22 & - & 2.40 & 0 & 0.952 & $2.66 \cdot 10^{16}$ \\
\hline${ }^{*} \mathrm{LiNbO}_{3}: \mathrm{Zn}(6.50)$ & 3.950 & 7.721 & 0.48 & - & 1.35 & 0 & 0.973 & $4.00 \cdot 10^{16}$
\end{tabular}

При ме чан и е. * Для указанных кристаллов индикатриса ФИРС не развивается при $I \sim 6.29 \mathrm{~W} / \mathrm{cm}^{2}$.

ния интенсивностей полос поглощения с частотами 3480 и $3465 \mathrm{~cm}^{-1}[5,17]$.

ИК-спектры поглощения исследованных кристаллов $\mathrm{LiNbO}_{3}$ представлены на рис. 1. Полоса с частотой $3465 \mathrm{~cm}^{-1}$ соответствует валентным колебаниям атомов водорода в комплексном дефекте $\mathrm{Nb}_{\mathrm{Li}}-\mathrm{OH}$ и является характерной для структуры высокосовершенного кристалла стехиометрического состава, в котором атомы водорода локализуются только в одной позиции, расположенной на полярной оси $[5,18]$. В спектре такого высокосовершенного кристалла присутствует только одна узкая линия $\left(s=3.0 \mathrm{~cm}^{-1}\right)$ с частотой $3465 \mathrm{~cm}^{-1}[5,18]$. В спектре ИК-поглощения исследованного нами стехиометрического кристалла $\mathrm{LiNbO}_{3 \text { stoich }}\left(6.0 \mathrm{wt} \% \mathrm{~K}_{2} \mathrm{O}\right)$, присутствуют три линии с частотами $3465,3480,3488 \mathrm{~cm}^{-1}$, что свидетельствует о трех позициях атомов водорода в этом кристалле. Для идеального стехиометрического кристалла, в котором концентрация дефектов $\mathrm{Nb}_{\mathrm{Li}}$ равна нулю, должна быть равна нулю и интенсивность линии с частотой $3480 \mathrm{~cm}^{-1}$. Линия с частотой $3480 \mathrm{~cm}^{-1}$ соответствует валентным колебаниям атомов водорода в дефектном комплексе $\mathrm{V}_{\mathrm{Li}} \mathrm{OH}$ и является характерной для кристалла конгруэнтного состава $(R=0.946)$ [19]. Отношение интенсивностей линий с частотами 3480 и $3465 \mathrm{~cm}^{-1}\left(I_{r e l}=I_{3480} / I_{3465}\right)$ при переходе от конгруэнтного кристалла к стехиометрическому практически линейно уменьшается с увеличением величины $R=\mathrm{Li} / \mathrm{Nb}[20,21]$. Рассчитанные значения величин $\mathrm{Li} / \mathrm{Nb}$ представлены в таблице.

На рис. $5(a-b)$ приведена зависимость интенсивности линии с частотой $3480 \mathrm{~cm}^{-1}$ и рассчитанной нами концентрации дефектов $\mathrm{Nb}_{\mathrm{Li}}$ от состава исследованных кристаллов $\mathrm{LiNbO}_{3}$. Видно, что концентрационное по- ведение интенсивности линии с частотой $3480 \mathrm{~cm}^{-1}$ хорошо коррелирует с рассчитанным количеством дефектов $\mathrm{Nb}_{\mathrm{Li}}$ вне зависимости от состава кристалла, за исключением сильнолегированных кристаллов $\mathrm{LiNbO}_{3}: \mathrm{Zn}(4.46-6.50 \mathrm{~mol} \%$ $\mathrm{ZnO})$. Из рис. 5, $a, b$ видно также, что интенсивность линии с частотой $3480 \mathrm{~cm}^{-1}$ в спектре кристаллов $\mathrm{LiNbO}_{3}: \mathrm{Zn}(4.46-6.50$ mol.\% $\mathrm{ZnO})$ не равна нулю, что свидетельствует о наличии в структуре кристаллов дефектов $\mathrm{Nb}_{\mathrm{Li}}$. Этот результат подтверждает вывод работы [22], в которой по данным полнопрофильного рентгеноструктурного анализа показано, что в структуре сильно легированных кристаллов $\mathrm{LiNbO}_{3}: \mathrm{Zn}(4.76-5.19 \mathrm{~mol} \% \mathrm{ZnO})$ присутствуют дефекты $\mathrm{Nb}_{\mathrm{Li}}$ и не подтверждает данные работы [23], согласно которым в структуре сильно легированных кристаллов $\mathrm{LiNbO}_{3}: \mathrm{Zn}$ нет точечных дефектов $\mathrm{Nb}_{\mathrm{Li}}$. Таким образом, из полученных данных следует, что для кристалла $\mathrm{LiNbO}_{3 \text { cong }}$ и для слаболегированных кристаллов $\mathrm{LiNbO}_{3}: \mathrm{Zn}$ (0.004-2.01 mol.\% $\left.\mathrm{ZnO}\right)$, характеризующихся высоким содержанием дефектов $\mathrm{Nb}_{\mathrm{Li}}$ должен быть значительным поляронный вклад в общую проводимость.

Величину поляронного вклада в общую проводимость кристалла $\mathrm{LiNbO}_{3}$ можно оценить из спектров фотолюминесценции. Спектры фотолюминесценции кристаллов $\mathrm{LiNbO}_{3}$ разного состава определяются особенностями статистического распределения по октаэдрам $\mathrm{O}_{6}$ точечных дефектов $\mathrm{Nb}_{\mathrm{Li}}, \mathrm{V}_{\mathrm{Li}}$ и др. (центров свечения) и представляют собой широкие полосы с основными максимумами при 390, 440, 500, 565 и $620 \mathrm{~nm}$, рис. 2. Вклад в уширение полос может вносить беспорядок, обусловленный разным расстоянием $\mathrm{Nb}-\mathrm{O}\left(\mathrm{Nb}_{\mathrm{Li}}-\mathrm{O}\right)$ и вследствие разного расположения в кислородно-окта- 

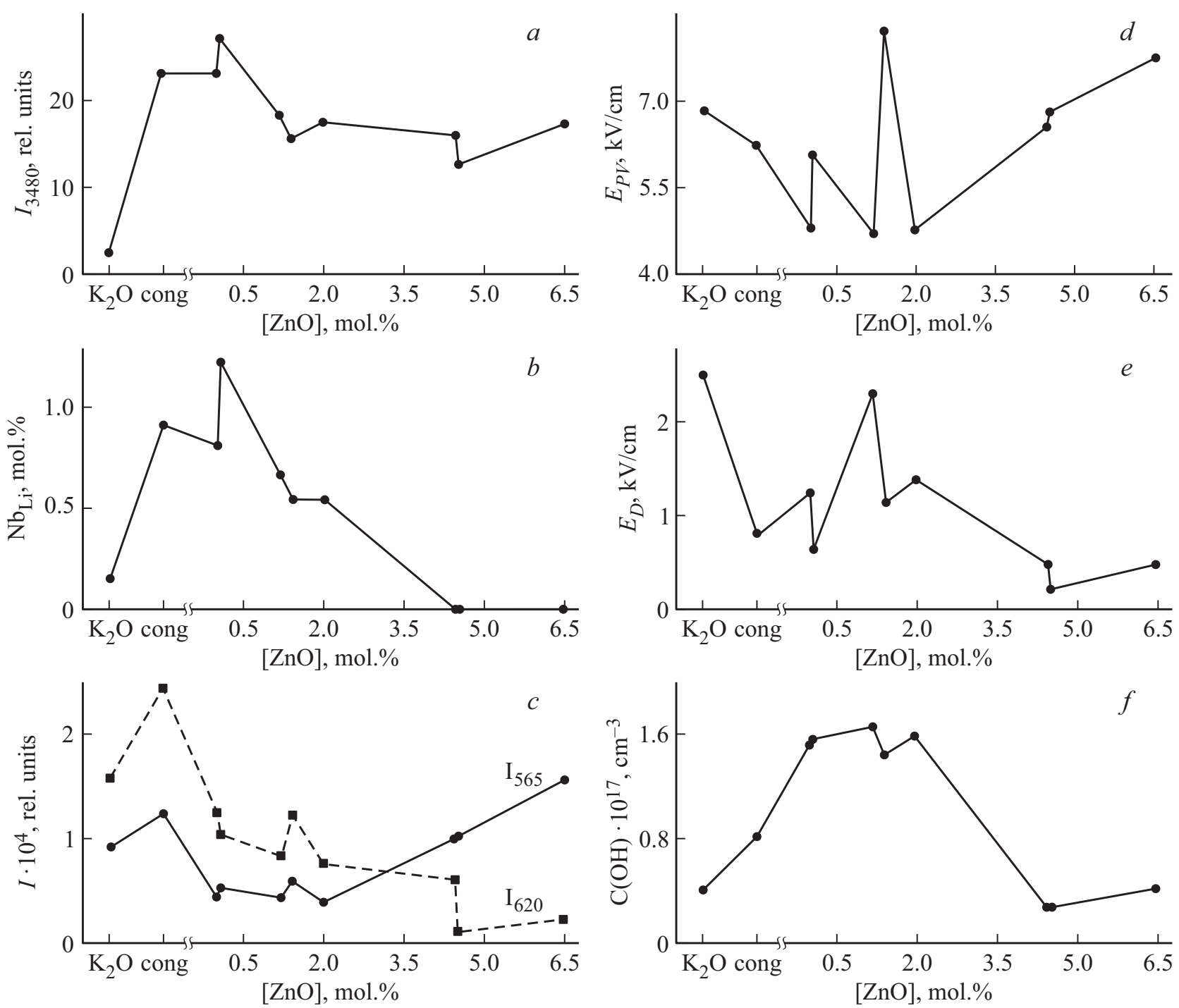

Рис. 5. Зависимости интенсивности линии с частотой $3480 \mathrm{~cm}^{-1}$ в спектре ИК-поглощения в области валентных колебаний $\mathrm{OH}^{-}$-групп $(a)$, концентрации точечных дефектов $\mathrm{Nb}_{\mathrm{Li}}(b)$, интенсивности люминесценции $\left(I_{565}\right.$ и $\left.I_{620}\right)$ центров свечения, связанных с дефектом $\mathrm{Nb}_{\mathrm{Li}}(c)$, фотовольтаического $(d)$ и диффузионного $(e)$ полей, концентрации ОН ${ }^{-}$-групп $(f)$ от концентрации цинка в исследуемых кристаллах $\mathrm{LiNbO}_{3}$.

эдрическом кластере $\mathrm{NbO}_{6}\left(\mathrm{Nb}_{\mathrm{Li}} \mathrm{O}_{6}\right)$ атомов водорода, связанных водородной связью с атомом кислорода. Из рис. 2 видно, что спектры фотолюминесценции кристаллов $\mathrm{LiNbO}_{3 \text { stoich }}\left(6.0 \mathrm{wt} . \% \mathrm{~K}_{2} \mathrm{O}\right), \mathrm{LiNbO}_{3 \text { cong }}$ и $\mathrm{LiNbO}_{3}: \mathrm{Zn}(0.004-6.50 \mathrm{~mol} . \% \mathrm{ZnO})$ состоят из широкого люминесцентного гало в видимой области спектра с максимумом при $600 \mathrm{~nm}(2.07 \mathrm{eV})$ для кристаллов $\mathrm{LiNbO}_{3 \text { stoich }}\left(6.0 \mathrm{wt} \% \mathrm{~K}_{2} \mathrm{O}\right), \mathrm{LiNbO}_{3 \text { cong }}$ и слаболегированных кристаллов $\mathrm{LiNbO}_{3}: \mathrm{Zn}(0.004,1.19,1.40$, $2.01 \mathrm{~mol} \% \mathrm{ZnO})$ и с максимумом при $500 \mathrm{~nm}(2.48 \mathrm{eV})$ для сильнолегированных кристаллов $\mathrm{LiNbO}_{3}: \mathrm{Zn}(4.46$, $4.54,6.50 \mathrm{~mol} \% \mathrm{ZnO})$. Каждый спектр состоит из набора элементарных полос излучения, природа которых обусловлена разными центрами свечения. Разделение спектров на составляющие по основным центрам свечения проводили на основании литературных данных:
$620-\mathrm{Nb}_{\mathrm{Nb}}-\mathrm{Nb}_{\mathrm{Li}}, 565-\mathrm{Nb}_{\mathrm{Li}}, 500-\mathrm{V}_{\mathrm{Li}}, 440-$ $\mathrm{Nb}_{\mathrm{Nb}}$ и $390 \mathrm{~nm}-\mathrm{V}_{\mathrm{Nb}}[12,24-26]$. Кроме того, в спектре кристаллов $\mathrm{LiNbO}_{3}: \mathrm{Zn}(4.46,4.54,6.50 \mathrm{~mol} \%$ ZnO наблюдается ряд дополнительных полос излучения при 460 и $710 \mathrm{~nm}$.

В кластере $\mathrm{NbO}_{6}$ атом $\mathrm{Nb}$ смещен вдоль полярной оси на $0.26 \AA$ из центросимметричного положения [4]. При этом существуют два разных расстояния между атомом $\mathrm{Nb}$ и шестью атомами О (2.112 и $1.889 \AA)$, рис. 4, что приводит к появлению отличного от нуля дипольного момента в направление полярной оси и наличию сегнетоэлектричества. Кроме того, кислородный октаэдр $\mathrm{NbO}_{6}$ по размерам меньше октаэдра $\mathrm{LiO}_{6}$, что, в том числе, определяет порядок вхождения легирующей примеси в решетку кристалла $\mathrm{LiNbO}_{3}$. Интенсивность излучения может зависеть от расстояния между 
атомами. Причем чем ближе атомы друг к другу и, возможно, чем менее искаженной является кислородная подрешетка, тем сильнее происходит тушение люминесценции. Так излучательная рекомбинация от пары $\mathrm{Nb}-\mathrm{O}$ в кластере $\mathrm{NbO}_{6}$ тушится за счет относительно малого расстояния между атомами $\mathrm{Nb}$ и $\mathrm{O}$, рис. 4. $\mathrm{C}$ другой стороны, образование дефекта $\mathrm{Nb}_{\mathrm{Li}}$ приводит к относительно большей интенсивности люминесценции в $\mathrm{LiNbO}_{3 \text { cong }}$ из-за большего размера кластера $\mathrm{Nb}_{\mathrm{Li}} \mathrm{O}_{6}$, рис. 4. Из рис. 2, $a$ видно, что наибольший вклад в фотолюминесценцию кристалла $\mathrm{LiNbO}_{3 \text { cong }}$ вносят центры свечения в виде глубоких электронных ловушек $\mathrm{Nb}_{\mathrm{Li}}-\mathrm{Nb}_{\mathrm{Nb}}$ с максимумом при $620 \mathrm{~nm}$ и $\mathrm{Nb}_{\mathrm{Li}}$ с максимумом при $565 \mathrm{~nm}$. Увеличение величины $R=\mathrm{Li} / \mathrm{Nb}$ приводит к тушению люминесцентного гало на $32 \%$, что говорит об уменьшении количества дефектов $\mathrm{Nb}_{\mathrm{Li}}$. Однако при этом люминесценция от центров свечения с участием дефектов $\mathrm{Nb}_{\mathrm{Li}}$ полностью не исчезает в кристалле $\mathrm{LiNbO}_{3 \text { stoich }}\left(6.0 \mathrm{wt} . \% \mathrm{~K}_{2} \mathrm{O}\right)$. Этот факт свидетельствует о наличии дефектов $\mathrm{Nb}_{\mathrm{Li}}$ в кристалле $\mathrm{LiNbO}_{3 \text { stoich }}\left(6.0 \mathrm{wt} . \% \mathrm{~K}_{2} \mathrm{O}\right)$ и убедительно подтверждает данные ИК-спектроскопии. Из рис. 1 видно, что интенсивность линии с частотой $3480 \mathrm{~cm}^{-1}$, являющейся характерной для кристалла конгруэнтного состава, в котором концентрация дефектов $\mathrm{Nb}_{\mathrm{Li}}$ максимальна, не равна нулю в спектре кристалла $\mathrm{LiNbO}_{3 \text { stoich }}\left(6.0\right.$ wt.\% $\left.\mathrm{K}_{2} \mathrm{O}\right)$.

На особенности электронной релаксации в зонной структуре $\mathrm{LiNbO}_{3}$ разного состава и, соответственно, на особенности фотолюминесценции кристалла $\mathrm{LiNbO}_{3}$ может оказывать заметное влияние водородная связь. Наличие водородных связей приводит к существенному изменению волновых функций внешних электронных орбиталей ионов кислорода и параметров его электронной поляризуемости, а также к заметному искажению кислородно-октаэдрических кластеров $\mathrm{NbO}_{6}$, определяющих электрооптические и люминесцентные свойства кристалла $\mathrm{LiNbO}_{3}$ [27,28]. Концентрация $\mathrm{OH}^{-}$-групп, рассчитанная из спектра ИК-поглощения по методу Клавира [11], в исследованных кристаллах $\mathrm{LiNbO}_{3}$ дана в таблице. Видно, что минимальная концентрация $\mathrm{OH}^{-}$-групп, как и минимальная концентрация дефектов $\mathrm{Nb}_{\mathrm{Li}}$, наблюдается в кристалле $\mathrm{LiNbO}_{3 \text { stoich }}\left(6.0 \mathrm{wt} \% \mathrm{~K}_{2} \mathrm{O}\right)$ и в сильно легированных кристаллах $\mathrm{LiNbO}_{3}: \mathrm{Zn}(4.46-6.50 \mathrm{~mol} . \% \mathrm{ZnO})$. Можно предположить, что уменьшение концентрации $\mathrm{OH}^{-}$-групп, входящих в состав комплексных дефектов, будет приводить к увеличению количества междоузельных протонов $\mathrm{H}^{+}$, что может обусловливать более высокую протонную проводимость и более эффективную излучательную рекомбинацию в видимой области спектра с их участием в качестве множественных мелких акцепторных уровней. При этом будет уменьшаться электронный вклад в электрическую проводимость [7].

В то же время, из рис. 5, $b$ и таблицы видно, что для исследованных кристаллов не наблюдается строгой корреляции между величиной $R=\mathrm{Li} / \mathrm{Nb}$ и концентрацией $\mathrm{OH}^{-}$-групп. Это может быть обусловлено тем, что величина протонной проводимости (при одинаковой концентрации гидроксильных $\mathrm{OH}^{-}$-групп в кристалле) определяется энергией связи О-Н. Для разных атомов водорода, локализованных в структуре кристалла в составе комплексных дефектов в разных позициях, она различна. Действительно в спектре ИК-поглощения кристаллов в области валентных колебаний $\mathrm{OH}^{-}$-групп наблюдается несколько линий (рис. 1), что свидетельствует о разных квазиупругих постоянных О-Н-связей в комплексных дефектах различного вида. Причем, различия в частотах валентных колебаний $\mathrm{OH}^{-}$-групп для линий одной природы, в спектрах кристаллов разного состава, могут достигать $50 \mathrm{~cm}^{-1}$, рис. 1 . Наличие разных позиций атомов водорода в структуре кристалла $\mathrm{LiNbO}_{3}$ может привести к изменению положения энергетических уровней в запрещенной зоне, что, по-видимому, может проявиться в спектрах фотолюминесценции.

Из рис. 2, $a$ и рис. 5, $c$ видно, что внедрение малых концентраций цинка $([\mathrm{ZnO}]<2.00 \mathrm{~mol} \%$ \%) в структуру конгруэнтного кристалла $\mathrm{LiNbO}_{3}$ сопровождается снижением излучательной рекомбинации дефектов $\mathrm{Nb}_{\mathrm{Li}}$. Этот факт можно объяснить плавным вытеснением цинком дефектов $\mathrm{Nb}_{\mathrm{Li}}$ с одновременным уменьшением компенсационных дефектов $\left(\mathrm{V}_{\mathrm{Li}}\right.$ и $\left.\mathrm{V}_{\mathrm{Nb}}\right)$ в структуре кристалла $\mathrm{LiNbO}_{3}$. Интересно отметить и тот факт, что в спектрах фотолюминесценции кристаллов $\mathrm{LiNbO}_{3}: \mathrm{Zn}(0.004-2.01 \mathrm{~mol} \% \mathrm{ZnO})$ не возникает полос излучения, связанных с дефектами $\mathrm{Zn}_{\mathrm{Nb}}, \mathrm{Zn}_{\mathrm{Li}}$ и др., обусловленных наличием примесных ионов $\mathrm{Zn}^{2+}$ в структуре кристалла. Такая особенность говорит об образовании в запрещенной зоне, вблизи дна зоны проводимости, множества мелких уровней энергий, так называемых „уровней прилипания“ [29], что должно увеличивать фотопроводимость кристаллов и, как следствие, уменьшать фоторефрактивный эффект.

Дальнейшее увеличение концентрации цинка в структуре кристалла $\mathrm{LiNbO}_{3}$ приводит к смещению люминесцентного гало на $\sim 100 \mathrm{~nm}$ за счет образования новых каналов рекомбинации с участием дефектов, обусловленных цинком. Из рис. 2, $b$ видно, что центры свечения, обусловленные наличием дефектов $\mathrm{Nb}_{\mathrm{Li}}$ (максимум при $565 \mathrm{~nm}$ ), присутствуют в спектрах сильно легированных кристаллов $\mathrm{LiNbO}_{3}: \mathrm{Zn}(4.46-6.50 \mathrm{~mol} \% \mathrm{ZnO})$. Этот факт, как и данные ИК-спектроскопии, противоречит точке зрения, высказанной в работе [23], согласно которой в сильно легированных кристаллах отсутствуют дефекты $\mathrm{Nb}_{\mathrm{Li}}$. Вероятно, наблюдаемые авторами [23] эффекты, которые они отнесли к изменениям за счет уменышения в кристалле концентрации дефектов $\mathrm{Nb}_{\mathrm{Li}}$, обусловлены изменением механизма внедрения $\mathrm{Zn}$ в структуру кристалла $\mathrm{LiNbO}_{3}$.

Для понимания процессов переноса фотоиндуцированных зарядов в кристаллах $\mathrm{LiNbO}_{3}$ разного состава и захвата их на ловушках различного уровня есть необходимость исследовать влияние $\mathrm{OH}^{-}$-групп на фотоиндуцированное рассеяние света и фоторефрактивные характеристики исследуемых кристаллов. Картины ФИРС 
кристаллов $\mathrm{LiNbO}_{3 \text { stoich }}\left(6.0 \mathrm{wt} \% \mathrm{~K}_{2} \mathrm{O}\right), \quad \mathrm{LiNbO}_{3 \text { cong }} \quad$ и $\mathrm{LiNbO}_{3}: \mathrm{Zn}(0.004-6.5 \mathrm{~mol} \% \mathrm{ZnO})$ приведены на рис. 3. В таблице приведены значения фотовольтаического $\left(E_{P V}\right)$ и диффузионного $\left(E_{D}\right)$ полей, а также ширины запрещенной зоны исследованных кристаллов $\mathrm{LiNbO}_{3}$, рассчитанные из характеристик индикатрисы спеклструктуры ФИРС по методике, изложенной в работе [12]. Видно, что наибольшими фотовольтаическими полями обладают кристаллы $\mathrm{LiNbO}_{3 \text { cong, }} \mathrm{LiNbO}_{3}: \mathrm{Zn}(0.07$, $1.40 \mathrm{~mol} \% \mathrm{ZnO})$ и сильно легированные кристаллы $\mathrm{LiNbO}_{3}: \mathrm{Zn}(4.46,4.54,6.50 \mathrm{~mol} \% \mathrm{ZnO})$. Для кристалла $\mathrm{LiNbO}_{3 \text { stoich }}\left(6.0 \mathrm{wt} \% \mathrm{~K}_{2} \mathrm{O}\right)$ и слаболегированных кристаллов $\mathrm{LiNbO}_{3}: \mathrm{Zn}(0.004,1.19,1.40,2.01 \mathrm{~mol} \% \mathrm{ZnO})$ характерно более высокое значение напряженности диффузионного поля и меньшее значение ширины запрещенной зоны по сравнению с кристаллами других составов, таблицы. Обнаруженные отличия в значениях фотополей могут быть обусловлены особенностями сложной дефектной структуры исследованных кристаллов $\mathrm{LiNbO}_{3}$. Разнообразие дефектов и особенности взаимодействий между структурными единицами в гетеродесмическом кристалле $\mathrm{LiNbO}_{3}$ должны приводить к появлению множества локализованных уровней энергий в запрещенной зоне, расположение которых определяется составом и тонкими особенностями упорядочения структурных единиц кристалла. При этом наблюдается корреляция в концентрационном поведении дефектов $\mathrm{Nb}_{\mathrm{Li}}$, интенсивности полос излучения, обусловленных дефектами $\mathrm{Nb}_{\mathrm{Li}}$ и величины напряженности фотовольтаического поля.

На рис. 5, $c-e$ представлены зависимости напряженности фотовольтаического поля и интенсивности полос излучения $\left(I_{565}\right.$ и $\left.I_{620}\right)$, обусловленных дефектами $\mathrm{Nb}_{\mathrm{Li}}$, от концентрации цинка в структуре кристалла $\mathrm{LiNbO}_{3}$. Видно, что в области концентраций от 0-1.19 mol.\% ZnO происходит уменьшение фотовольтаического поля и уменьшение интенсивности свечения, обусловленной дефектами $\mathrm{Nb}_{\mathrm{Li}}$. Последний факт свидетельствует об уменьшении количества глубоких ловушек захвата электронов $\mathrm{Nb}_{\mathrm{Li}}$. Но, поскольку преимущественным механизмом фоторефракции в кристалле $\mathrm{LiNbO}_{3}$ является фотовольтаический [4], то в области от 0 до $1.19 \mathrm{~mol}$ \% $\mathrm{ZnO}$ должно происходить образование мелких донорных уровней у дна зоны проводимости, что увеличивают фотопроводимость кристалла. Этот вывод подтверждается результатами работы [23], в которой показано, что увеличение концентрации цинка в кристалле $\mathrm{LiNbO}_{3}$ приводит к увеличению фотопроводимости. Кроме того, в данных кристаллах по сравнению с кристал-

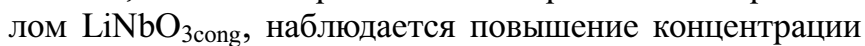
гидроксильных групп (таблица), что может влиять на изменение поляризуемости кислородно-октаэдрических кластеров $\mathrm{MeO}_{6}$.

Из рис. $5, c, d$ видно также, что увеличение концентрации с 2.01 до $6.5 \mathrm{~mol} \% \mathrm{ZnO}$ в кристалле $\mathrm{LiNbO}_{3}$ приводит к увеличению величины напряженности фотовольтаического поля $E_{P V}$ и интенсивности максимума люминесценции при $565 \mathrm{~nm}\left(I_{565}\right)$, а также к снижению интенсивности максимума люминесценции при $620 \mathrm{~nm}$ $\left(I_{620}\right)$, обусловленных, соответственно, дефектами $\mathrm{Nb}_{\mathrm{Li}}$ и биполяроном $\mathrm{Nb}_{\mathrm{Li}}-\mathrm{Nb}_{\mathrm{Nb}}$. Согласно работе [23] увеличение $E_{P V}$ можно объяснить с точки зрения увеличения значения фотовольтаической постоянной $k \alpha$ в области концентрации $3<\mathrm{Zn}<5$ at.\%. Однако, фотопроводимость в данной концентрационной области растет по гиперболическому закону [30]. При этом происходит разрушение биполяронной пары $\mathrm{Nb}_{\mathrm{Li}}-\mathrm{Nb}_{\mathrm{Nb}}$ за счет увеличения концентрации дефектов $\mathrm{Zn}_{\mathrm{Li}}$. В структуре кристалла $\mathrm{LiNbO}_{3}$ цинк может вытеснять не только дефекты $\mathrm{Nb}_{\mathrm{Li}}$, но и литий из основных позиций, образуя дефекты $\mathrm{Zn}_{\mathrm{Li}}$, что приводит к увеличению количества глубоких дырочных центров захвата в виде дефектов $\mathrm{V}_{\mathrm{Li}}$ с энергией $E=-0.44 \mathrm{eV}[16]$. С другой стороны, увеличение интенсивности максимума люминесценции при $565 \mathrm{~nm}$ можно объяснить также внедрением катионов цинка в ниобиевый октаэдр с образованием дефектов $\mathrm{Zn}_{\mathrm{Nb}}$ и одновременным уменьшением концентрации дефектов $\mathrm{Nb}_{\mathrm{Li}}$. Однако, согласно данным работы [23], это возможно только при концентрациях $\mathrm{ZnO}>7.00$ mol.\%. Кроме того, в таких столь сильно легированных кристаллах наблюдается существенно более низкое по сравнению с другими кристаллами содержание $\mathrm{OH}^{-}$-групп, а также увеличение ширины запрещенной зоны на $0.3-0.4 \mathrm{eV}$, таблица. Таким образом, можно сделать вывод, что с повышением концентрации цинка в сильно легированных кристаллах $\mathrm{LiNbO}_{3}: \mathrm{Zn}$ происходит несколько параллельных процессов, приводящих к уменьшению эффекта фоторефракции: увеличение фотопроводимости $n$ - и $p$-типа за счет увеличения концентрации цинка и увеличения числа межузельных протонов $\mathrm{H}^{+}$, уменьшения числа центров захвата в виде биполяронной пары $\mathrm{Nb}_{\mathrm{Li}}-\mathrm{Nb}_{\mathrm{Nb}}$ с увеличением свечения от полярона малого радиуса $\mathrm{Nb}_{\mathrm{Li}}$.

\section{4. Заключение}

Таким образом, в сильно легированных кристаллах $\mathrm{LiNbO}_{3}: \mathrm{Zn}(4.46-6.50 \mathrm{~mol} \%$ Z ZnO) обнаружено заметное уменьшение концентрации $\mathrm{OH}^{-}$-групп и увеличение концентрации междоузельных протонов $\mathrm{H}^{+}$, напряженности фотовольтаического поля, ширины запрещенной зоны и излучательной рекомбинации в зеленой области спектра. Для слабо легированных кристаллов $\mathrm{LiNbO}_{3}: \mathrm{Zn}(0.004-2.01 \mathrm{~mol} \% \mathrm{ZnO})$ увеличение концентрации $\mathrm{OH}^{-}$-групп и снижение люминесценции от центров свечения с участием $\mathrm{Nb}_{\mathrm{Li}}$ приводит к уменьшению величины напряженности диффузионного и фотовольтаического полей за счет образования мелких энергетических уровней энергии в близи дна зоны проводимости. Кроме того, величина протонной проводимости в кристалле $\mathrm{LiNbO}_{3}$ зависит от особенностей локализации атомов водорода в его структуре и различна для кристаллов разного состава. В стехиометрическом кристалле $\mathrm{LiNbO}_{3 \text { stoich }}\left(6.0 \mathrm{wt} \% \mathrm{~K}_{2} \mathrm{O}\right)$ атомы водорода об- 
разуют водородные связи преимущественно с одним из атомов кислорода кислородно-октаэдрического кластера $\mathrm{NbO}_{6}$, что приводит к их заметному искажению, снижению ширины запрещенной зоны, увеличению вклада в протонную проводимость и уменьшению излучательной рекомбинации центров свечения $\mathrm{Nb}_{\mathrm{Nb}}$ и $\mathrm{Nb}_{\mathrm{Li}}$. В структуре кристаллов $\mathrm{LiNbO}_{3}: \mathrm{Zn}$ атомы водорода входят в состав комплексных дефектов $M e-\mathrm{OH}$ и $M e-\mathrm{OH}-M e\left(\mathrm{~V}_{\mathrm{Li}}\right)$ $(M e$ - примесный $(\mathrm{Zn})$ или основной $(\mathrm{Li}, \mathrm{Nb})$ катион) и не оказывают такого заметного влияния на кислороднооктаэдрические кластеры $\mathrm{NbO}_{6}$, ширину запрещенной зоны и протонную проводимость, как в стехиометрическом кристалле. Рассчитанная в работе концентрационная зависимость дефектов $\mathrm{Nb}_{\mathrm{Li}}$ коррелирует с концентрационной зависимостью интенсивности линии с частотой $\sim 3480 \mathrm{~cm}^{-1}$ спектра ИК-поглощения. Этот факт позволяет оценить концентрацию точечных дефектов $\mathrm{Nb}_{\mathrm{Li}}$ в кристаллах ниобата лития разного состава.

\section{Благодарности}

Авторы выражают благодарность В.Б. Пикулеву за помощь в постановке эксперимента по фотолюминесценции и обсуждение экспериментальных данных.

\section{Финансирование работы}

Работа выполнена при частичной финансовой поддержке РФФИ, грант № 20-33-90078.

\section{Конфликт интересов}

Авторы заявляют об отсутствии у них конфликта интересов.

\section{Список литературы}

[1] Ю.С. Кузьминов. Электрооптический и нелинейно-оптический кристалл ниобата лития. Наука, М. (1987). C. 9-24.

[2] K.K. Wong. Properties of Lithium Niobate. INSPEC-The institution of Electrical Engineers. London, UK (2002). 143 p.

[3] L. Arizmendi. Phys. Status Solidi A 201, 2, 253 (2004).

[4] Н.В. Сидоров, Т.Р. Волк, Б.Н. Маврин, В.Т. Калинников. Ниобат лития: дефекты, фоторефракция, колебательный спектр, поляритоны. Наука, М. (2003) С. 13-56.

[5] K. Lengyel, A. Peter, L. Kovacs, G. Corradi, L. Palfavi, J. Hebling, M. Unferdorben, G. Dravecz, I. Hajdara, Zs. Szaller, K. Polgar. Appl. Phys. Rev. 2, 4, 040601 (2015).

[6] E. Kratzig. Ferroelectrics 21, 1, 635 (1978).

[7] F. Jermann, M. Simon, E. Kratzig. J. Opt. Soc. Am. B 12, 11, 2066 (1995).

[8] D.A. Bryan, Robert Gerson, H.E. Tomaschke. Appl. Phys. Lett. 44, 9, 847 (1984).

[9] М.Н. Палатников, Н.В. Сидоров, О.В. Макарова, И.В. Бирюкова. Фундаментальные аспекты технологии сильно легированных кристаллов ниобата лития. КНЦ РАН, Апатиты (2017). 241 с.
[10] M.N. Palatnikov, N.V. Sidorov. Oxide electronics and functional properties of transition metal oxides. NOVA Science Publichers, USA (2014). P. 31-168.

[11] S. Klauer, M. Wöhlecke, S. Kapphan. Phys. Rev. B 45, 6, 2786 (1992).

[12] M. Goulkov, M. Imlau, Th. Woike. Phys. Rev. B 77, 23, 235110 (2008).

[13] И.Ш. Ахмадуллин, В.А. Голенищев-Кутузов, С.А. Мигачев. ФТT 40, 6, 1109 (1998).

[14] O. Schirmer, O. Thiemann, M. Wöhlecke. J. Phys. Chem. Solids 52, 1, 185 (1991).

[15] A.P. Wilkinson, A.K. Cheerham, R.H. Jarman. J. Appl. Phys. 74, 5, 3080 (1993).

[16] H.J. Donnerberg, S.M. Tomlinson, C.R.A. Catlow. J. Phys. Chem. Solids 52, 1, 201 (1991).

[17] М.И. Саллум, О.С. Грунский, А.А. Маньшина, А.С. Тверьянович, Ю.С. Тверьянович. Изв. РАН. Сер. хим. 73, 11, 2162 (2009).

[18] K. Polgar, A. Peter, L. Kovacs, G. Corradi, Zs. Szaller. J. Cryst. Growth 177, 3-4, 211 (1997).

[19] N. Iyi, K. Kitamura, F. Izumi, J.K. Yamamoto, T. Hayashi, H. Asano, S. Kimura. Chem J. Solid Status. 101, 2, 340 (1992).

[20] G. Dravecz, L. Kovács. Appl. Phys. B 88, 2, 305 (2007).

[21] G. Dravecz, L. Kovács, A. Peter, K. Polgar, P. Bourson. Phys. Status Solidi C 4, 3, 1313 (2007).

[22] Н.В. Сидоров, Н.А. Теплякова, А.А. Яничев, М.Н. Палатников, О.В. Макарова, Л.А. Алешина, А.В. Кадетова. Неорган. материалы 53, 5, 491 (2017).

[23] T. Volk, B. Maximov, T. Chernaya, N. Rubinina, M. Wöhlecke, V. Simonov. Appl. Phys. B 72, 6, 647 (2001).

[24] В.Ю. Яковлев, Е.В. Кабанова, Т. Вебер, П. Пауфлер. ФТТ 43, 8,1520 (2001).

[25] D.M. Krol, G. Blasse, R.C. Powell. J. Chem. Phys. 73, 1, 163 (1980).

[26] C. Fischer, M. Wöhlecke, T. Volk, N. Rubinina. Phys. Status Solidi A 137, 1, 247 (1993).

[27] С.В. Евдокимов, А.В. Яценко. Кристаллография 48, 4, 594 (2003).

[28] J.M. Cabrera, J. Olivares, M. Carrascosa, J. Rams, R. Müller, E. Déguez. Adv. Phys. 45, 5, 349 (1996).

[29] А.А. Блистанов, В.М. Любченко, А.Н. Горюнова. Кристаллография 43, 1, 86 (1998).

[30] T. Volk, M. Wöhlecke, N. Rubinina, A. Reichert, N. Razumovski. Ferroelectrics 183, 1, 291 (1996).

Редактор Т.Н. Василевская 